\title{
The luminosity function of individually undetectable stars
}

\author{
R.C. Snel \\ Lund Observatory, Box 43, S-221 00 Lund, Sweden \\ e-mail: Ralph.Snel@astro.lu.se
}

Received July 18; accepted September 29, 1997

\begin{abstract}
A method is presented to derive the faint end of the stellar luminosity function in crowded fields. The method assumes that two images that look similar, have similar luminosity functions. A simulated image with known luminosity function is compared with an observed image, and the luminosity function is adjusted until the images look sufficiently similar. A number of tests were performed on simulated and real images, showing the effects of various error sources. The histogram of the image was used to determine similarity. It was possible to derive the luminosity function to a limit of approximately one source per beam, with the beam defined as $\pi$ times the square of the half width at half maximum of the point spread function. Defining the limit of detection for classical photometry as the magnitude where the completeness of detection dropped below 0.5, and for the luminosity function derived with this method at the magnitude where the uncertainty in the luminosity function exceeded the derived number of stars at this magnitude, it was possible to derive the luminosity function up to two magnitudes fainter than possible with classical photometry. The accuracy was limited by knowledge of the analog-digital converter effects, read-out noise, and point spread function.
\end{abstract}

Key words: methods: data analysis - methods: observational - methods: statistical - techniques: photometric - stars: luminosity function, mass function

\section{Introduction}

Classical crowded field photometry detects and measures individual stars down to a limiting magnitude at which they can not be discerned from spurious noise peaks in the background of the image. Severe crowding limits detection of fainter stars measurable in an uncrowded field. The combined effect of the undetectable stars on the image is still measurable using statistical techniques.

Saha (1995) formulates a notion of detection significance (for uncrowded point sources) independent of any special choice of statistics for source detection.
Completeness and spurious detections are related and depend on the source brightness, the pixel size of the detector and the point spread function (PSF). Also for uncrowded sources, Zepka et al. (1994) describe a method using the histogram of the data and the statistical properties of the background. With their method, it is possible to detect a fraction of the objects that are well below five $\sigma$, but without detecting large numbers of spurious noise peaks.

The stellar luminosity function (LF) is defined as the number of stars per cubic parsec within an absolute magnitude interval. The initial mass function and star formation history of a sample of stars are reflected in the LF, which in itself is, given the distance to the stars, an easily accessible observational quantity. For the determination of the LF of an observed field of stars the actual detection of the stars is not strictly necessary, since no individual positions and intensities for the stars are needed. Only the number of stars as a function of magnitude is needed. The incompleteness of detection can partly be eliminated through estimation of the fraction of detected stars per magnitude interval. When this fraction becomes small, the uncertainty in the estimated LF gets large, and when the fraction is zero, the limit of the detection has been reached.

Figure 1 shows an extreme case, where the stars are so faint that none can be detected without detecting a large number of spurious noise peaks as well. It is not possible to say anything about the LF using the method described above. However, if one compares this noisy image with an image containing only noise, the images will not be statistically equivalent, as if drawn from the same statistical ensemble. All deviations in intensity, due to the undetectable stars, will be positive. If the number of stars is large, the net effect of all these small contributions can be significant, causing an increase in the apparent background level and a shape change (especially an increase in the width) of the noise distribution of the apparent background level (Fig. 2).

In classical crowded field photometry this net effect will contribute to the overall background in the image. Stetson (1987) suggests that the sky background could be calculated from the observed LF and PSF. Here, the 

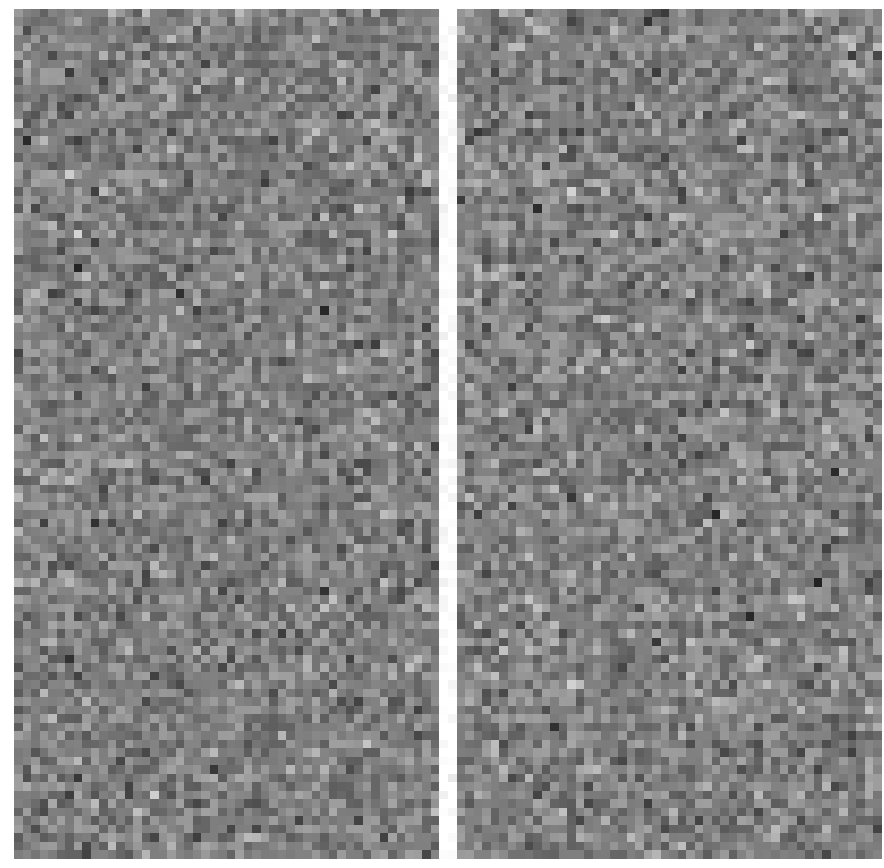

Fig. 1. Left: image showing pure Gaussian noise. Right: image showing pure Gaussian noise plus numerous faint stars. The noise has a $1 \sigma$ amplitude of 0.8 analog to digital units (ADU) The original image of $800 \times 800$ pixels contains 10000 equal magnitude stars, the sub-image shown here contains around 80 stars. The full width at half maximum of the PSF for the stars is 1.3 pixels, the maximum amplitude for a single star is 0.8 ADU

opposite is proposed: to derive the faint part of the LF using the properties of the noise and background in the image.

Comparing the appearance of the observed image with a simulated image with known LF, the LF can be adjusted to present statistically equivalent images, thus deriving an estimate for the LF. With this procedure, it is possible to derive the LF for stars that are up to two magnitudes fainter than those that can be detected as individual stars.

\section{Method used}

A new method is proposed for determining the faint end of the luminosity function: LUMINOUS (LUminosityfunction Modelling of Image Noise to Observe Undetectable Stars). The basic principle is that if two images look "similar" enough, the luminosity functions are similar.

A simulated image is created, using information derived from the observed image. This includes noise characteristics and sensitivity of the detector, analog to digital converter (ADC) effects, PSF, diffuse sky brightness, detected stars, and the location of cosmic ray effects (CREs) and defective pixels. Iteratively modifying the number of detected stars for certain magnitude intervals, the simulated image is made to look more like the observed image.

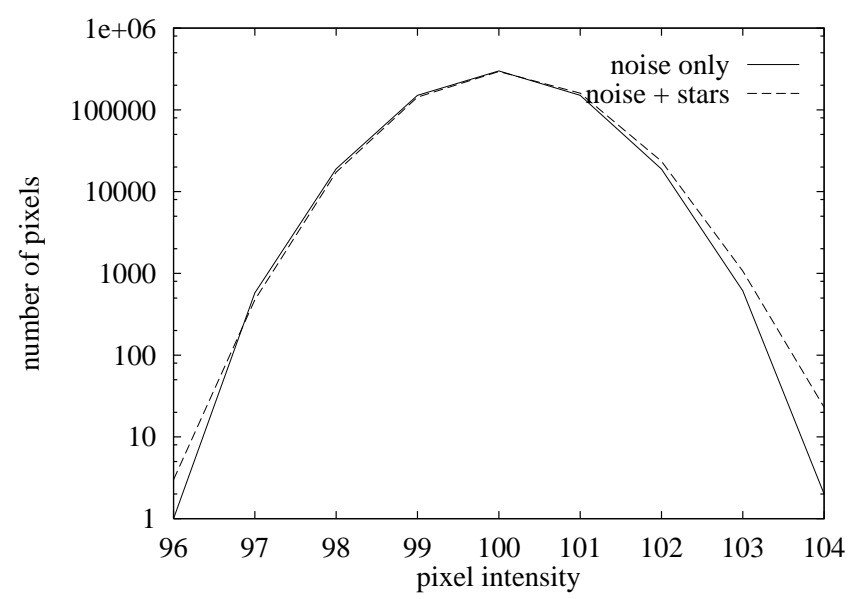

Fig. 2. Pixel intensity histograms of the images shown in Fig. 1. A 100 ADU bias level has been added to the images

Any additional stars needed to improve the appearance of the simulated image are located at random.

To determine the similarity between the images, the histograms of pixel intensities in the images are used. The histogram has the advantage of independence of the location of the stars in the image. A pixel-to-pixel comparison of the simulated and real image would be sensitive to the location of the (undetectable) faint stars.

\subsection{Measure of similarity}

The histogram of the simulated image is compared with that of the original image. The goodness of fit of the two histograms is expressed with the $\chi^{2}$ parameter, defined as

$\chi^{2}=\frac{1}{N} \sum_{i=i_{0}}^{i_{0}+N} \frac{\left(R_{i}-S_{i}\right)^{2}}{\left(R_{i}+S_{i}\right)}$,

where $i_{0}$ is the first significant intensity bin in the histograms, $N+1$ is the number of histogram bins over which $\chi^{2}$ is determined, and $R_{i}$ and $S_{i}$ are the counts in the $i^{\text {th }}$ bin of the two histograms.

This $\chi^{2}$ measure covers the interesting range of intensities, where the contribution from bright stars is small, but the largest effect from the undetectable and faint stars is located. Statistically equivalent histograms should have a $\chi^{2}$ of unity.

\subsection{What is being modelled?}

Since the aim of LUMINous is to detect stars that are of the same intensity as fluctuations in the noise of the image, this noise should be modelled as accurately as possible. In the original raw image, all intensities are in the form of integer values. Subsequent image processing will change these intensities to real values, more closely representing the true intensity of the light falling on the CCD pixels. This processing includes e.g. bias subtraction and flat fielding. 
With real values it is not obvious how to define a unique histogram. The properties of the noise are changed in a complex way. In the raw image the noise is easily defined as the quadratic sum of the read-out noise and the Poisson (shot) noise. As an example, consider a part of the image with a low value for the flat field. The noise in this part will be amplified during flat fielding of the image. If the flat field has a small systematic error, this will affect the intensities and give them a larger uncertainty. If, on the other hand, the true intensity of the image is modelled, and then multiplied with the flat field, the systematic error will affect the intensities as well. The Poisson noise in the intensities will add to the effect of this error, but since the value of the flat field was low, and the error small, the effect of the Poisson noise will dominate, and can easily be calculated.

For these reasons the raw image is modelled. Instead of correcting for effects of the detector, they are modelled. The method chosen here is to model the physical processes taking place. An LF dictates a certain distribution of stars projected on the detector. Spatial sensitivity variations of the detector are modelled through the flat field. The photons are converted to electrons, which are read out through electronics with a certain gain factor, linearity effects, dynamic range, read-out noise etc. Raw data is the final product. The following steps are taken when converting an input LF to a simulated histogram: add dark image, add known stars (from photometry), add unknown stars (from input LF), apply flat-field effects, add Poisson noise, add read-out noise, convert from electrons to ana$\log$ to digital unit (ADU), add bias level, add bias image, apply ADC nonlinearity effects, multiply with CRE mask, create histogram.

Careful mapping of the noise characteristics of the individual pixels of the image during processing could be done as an alternative, and should give the same results in the end, but the effects of the noise on the histogram would complicate matters and make comparison of two images through the histogram impractical.

\subsection{Assumptions}

The following assumptions have been made for the method described here:

- If two images appear similar, their LFs are similar.

- Only stars are present in the image.

Each object present in the image is defined through only three parameters: two spatial coordinates and an intensity. This means that extended objects like galaxies are not modelled. Nor are CREs modelled.

LUMINOUS is designed to work in crowded stellar fields. In all reasonably practical cases (e.g. globular clusters, nearby galaxies), the stars will dominate. Resolved galaxies can be identified, and excluded from the histogram. Unresolved galaxies are treated as single stars, but since their number is low, they will not affect the LF.
- The faint stars are distributed randomly over the image.

Clustering of stars is assumed to be purely random. Brighter stars are detected by conventional means, and can be modelled with appropriate position and intensity.

For sufficiently small fields of view, the assumption is valid. For globular clusters, a larger field of view may require modelling of the distribution of stars, e.g. adopting the over-all intensity profile of the cluster.

- The PSF is constant over the image.

Depending on e.g. the optics of the telescope, this assumption may not be true. Deviations from a constant PSF are usually largest at the edge of the field, so using a smaller part of the detector may reduce the problem.

- The distribution of photon intensities is subject to Poisson statistics.

- The read-out noise is normally distributed.

- The diffuse sky background is constant over the image. This assumption is easy to check by inspecting the image.

Not all of these assumptions are critical. If any of the assumptions is invalid, the corresponding effect should be included when creating the simulated image.

\subsection{Required information}

A number of properties of the image should be known before Luminous can be applied. These include:

\section{- The PSF.}

For classical PSF fitting photometry the PSF has to be determined. If the PSF is defined to a sufficiently large radius it can be used as-is. If not, some form of model PSF could be used.

- Read-out noise and gain (electrons per ADU) of the CCD.

- The bias level of the image.

- The bias image of the CCD.

- The flat field of the CCD.

Since LUMINOUS works with raw image data, the sensitivity of the CCD should be included in the model.

- The ADC effects.

The transformation from detected number of electrons to registered ADU is not completely linear. Nonlinearity effects of the ADC are modelled.

- The dark image and dark time.

Dark current and warm or hot pixels are modelled. The dark time is the time from the last clearing of the CCD to the time at read-out.

- The locations of pixels affected by CREs.

The signal of CREs can affect the histogram. The affected pixels can be excluded from the histogram of the image.

Much of this information is needed in standard image processing, and is usually known for the instrument used. The accuracy with which some of the effects are known is 


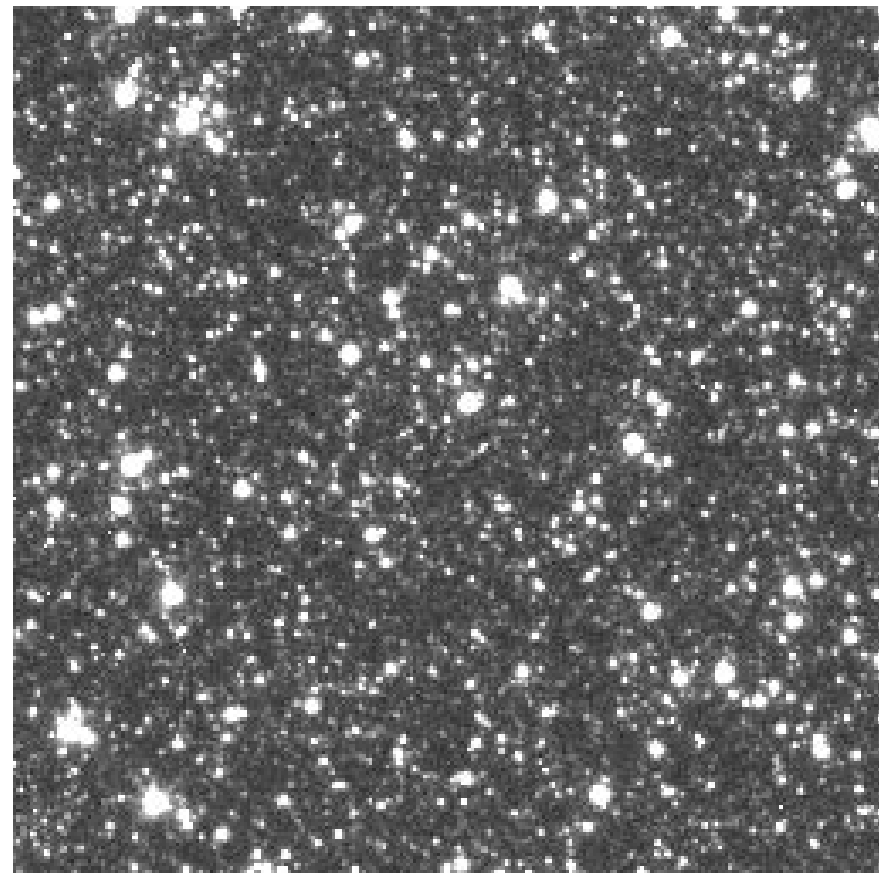

Fig. 3. $256 \times 256$ pixel sub-image of the $800 \times 800$ pixel simulated image. The LF for this image is given in Fig. 4

not always sufficient for LUMINOUS. For classical photometry the read-out noise is usually only used to determine the signal to noise ratio, and a low accuracy value suffices. For LUMINOUS it should be checked if the given value is correct and stable, since the statistical properties of the read-out noise are very important.

Except for the effects discussed, the CCD is considered perfect. This includes absence of effects like geometric distortion, deferred charge, charge transfer problems or trailing of the images, video noise, sub-pixel scale sensitivity variations, residual image effects, etc.

\section{Simulations}

To see how well Luminous works and which accuracies can be expected, several simulations were made. The simulations were chosen to resemble an exposure of a crowded stellar field with the Hubble Space Telescope (HST) on a single CCD of the Wide Field Camera (WFC) of the Wide Field Planetary Camera 2 (WFPC2).

An input LF with arbitrary magnitude offset (Fig. 4) was used to generate a simulated test image, shown in Fig. 3. This image was then analysed with DAOPHOT (Stetson 1987) and the completeness of detection was calculated as a function of magnitude. At magnitude 20 the completeness was still near unity, and dropped off to reach zero at magnitude 23 , passing 0.5 at 21.5. The LF of the detected stars (detected LF hereafter) is shown in Fig. 4.

The LF and diffuse background level were derived with LUMINOUS (derived LF hereafter), using the histogram and true PSF of the image. All counts for magnitudes

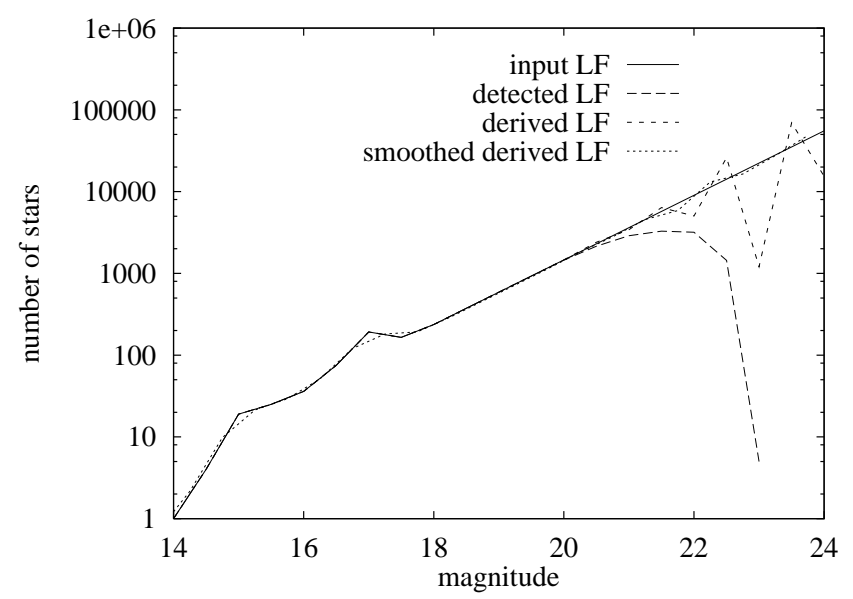

Fig. 4. Input, detected, derived and smoothed derived LF for the simulation

of 20 and higher (up to 24 , in 0.5 magnitude increments) could be adjusted.

\subsection{Possible error sources}

A first test was done to see if Luminous would work with no other errors than those due to noise. All parameters describing the LF were left to vary freely, but values for the bias level, read-out noise, shape of the PSF, flat field, etc., were unchanged, using the same values as used to create the test image.

Figure 4 shows that the derived LF was not identical to the input one. All deviations result from noise effects and limitations of Luminous. The large fluctuations in the faint part of the LF were the result of noise combined with the fact that few histogram bins could be used to derive the number of stars in these faint magnitude bins. The derived LF fluctuated around the true LF curve, the residuals being alternately positive and negative. When the derived LF was smoothed, the result looked much more acceptable. The goodness of the fit was not significantly affected (an increase in $\chi^{2}$ from $0.995 \pm 0.017$ to $1.018 \pm 0.026)$, since the numbers of stars in adjacent bins were strongly correlated with negative correlation factors.

The smoothing applied was an interpolation. Each point was replaced by the average of the magnitude of two neighbouring points, with the ordinate the intensity weighted counts of the neighbouring points. The smoothing can be omitted, but the counts in adjacent bins have large correlation coefficients, and the results have to be interpreted accordingly.

The uncertainty in the number of stars grew roughly exponentially with magnitude. For bright stars the slope was lower, resulting in uncertainty of the same order of magnitude as the value of the LF, thus defining a lower limit on the magnitude range for this method. For faint 


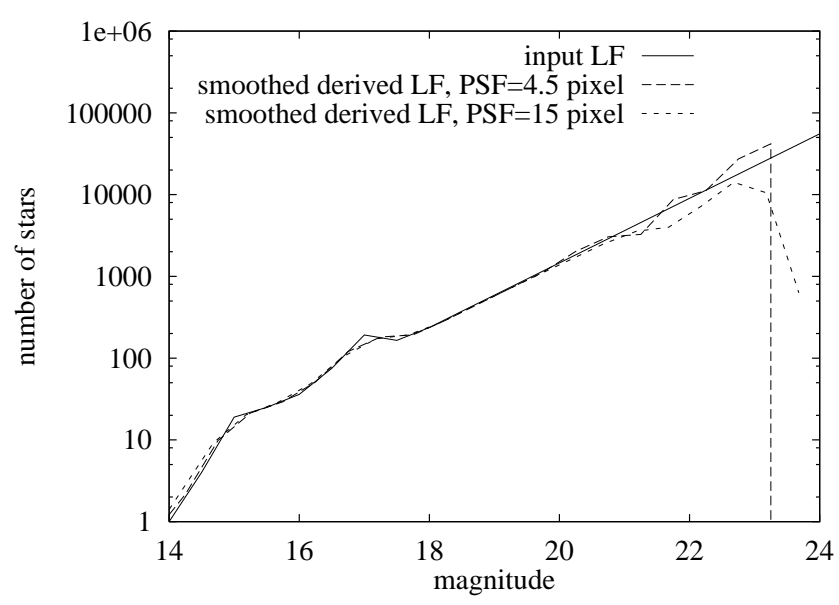

Fig. 5. Input and smoothed derived LFs for PSFs with radii 4.5 and 15 pixels, derived from the image. The FWHM of the PSF is approximately 1.5 pixels

stars, the uncertainty in the number of stars grew approximately with $\mathrm{e}^{\mathrm{mag} * 1.5}$, whereas the $\mathrm{LF}$ grew with $\mathrm{e}^{\mathrm{mag} * 0.9}$.

To investigate the effects of some of the fixed parameters these were changed by a small amount where possible, and the effect on the LF was studied.

\subsubsection{Bias image}

The effect of the bias image is to add an "intensity" offset to the individual pixels of the image, without affecting the noise of the intensities of these pixels. A true intensity (like diffuse sky background) would affect the noise. If there are errors in the bias image, the effect on the histogram of the image can be approximated as having two components: a shift in the entire bias level of the image (resulting in an identical shift of the histogram along the intensity axis), and an increase in the read-out noise. The errors in the bias image will effectively cause a widening of the distribution of pixel intensities around the true bias level. Both effects, a change in bias level and a change in read-out noise, are covered below.

\subsubsection{Bias level}

Small errors in the bias level are to be expected. The effect is compensated for by the diffuse background level parameter. The additional intensity alters the noise properties of the simulated image (through Poisson noise), which in turn affects the derived number of stars for the faintest magnitudes. An increase of 1 ADU in the bias level caused the derived LF to contain twice the number of stars for magnitude 24.0, and about 70 percent of the number of stars for magnitude 22.5 .

\subsubsection{Read-out noise}

An increase in the read-out noise from 5.0 to 5.5 electrons caused a change in the width of the peak in the histogram around the bias level. There was no obvious way to take this into account changing the background level or number of stars. For the very faintest pixels of the image, the slope in the histogram was not correct, and could not be corrected. On the bright side of the peak, corrections could be made changing appropriate parameters. $\chi^{2}$ was not as low as without the read-out noise error. The effects on the derived LF were similar as for the error in the bias level.

\subsubsection{Errors in the PSF}

The code to generate the simulated image uses a constant radius for the PSF. In realistic cases, the PSF is extracted from the image, and may contain undetected faint stars. A large PSF radius has the advantage of taking into account the wings of the PSF, including relatively bright diffraction spikes, but has the disadvantage of including undetectable stars, which can affect the fainter part of the derived LF. Figure 5 shows the derived LFs, with PSFs determined from the image, defined over a radius of 15 pixels (approximately 10 times the full width at half maximum, FWHM, of the PSF) and 4.5 pixels. For the PSF with large radius, there is a significant dip around magnitude 22 , and the total number of stars is underestimated over a large range of magnitudes.

With a smaller PSF radius, the number of undetected stars in the PSF decreased, but so did the influence of the wings. Two effects could be expected: a decrease of the derived number of faint stars due to extra stars in the PSF, and an increase of faint stars to account for missing intensity in the truncated wings of brighter stars. For a

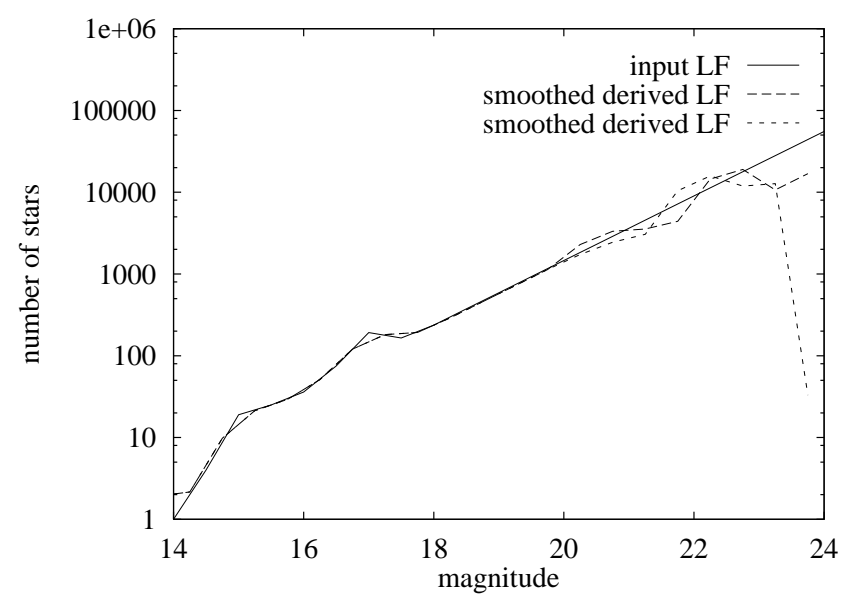

Fig. 6. Input and derived LFs for a simulation with errors in the ADC effects. The two curves for the derived LF are two solutions with very similar $\chi^{2}$

PSF radius of 4.5 pixels, the derived LF shows a slight increase in slope compared to the input LF, but the derived number of stars is more realistic than for the PSF with the large radius. 


\subsubsection{ADC effects}

Analog to digital converter effects are normally not particularly important for photometry. The effects are very small deviations from linearity between electrons in the CCD and measured ADU. For the histogram, the effects become more important. The number of pixels in one bin may be over-represented, while under-represented in an adjacent bin. The ADC effects have a similar influence on the histogram as flat field effects have on a CCD image. An error in the ADC effects can show up in the derived LF as a large gradient. The error can partially be eliminated by a change in the number of stars for two adjacent magnitude bins, increasing the one, while decreasing the other. The fit to the histogram of the observed image will be improved at the expense of a systematic error in the derived LF. This can also happen for relatively bright stars, depending on where exactly in the histogram the largest parts of the ADC effects are located.

An error in the ADC effects was introduced by shifting the entire observed histogram along the intensity axis, and adding the same intensity to the bias level in the model. Figure 6 shows two smoothed LFs, derived with the ADC effects error in the model. The best values for $\chi^{2}$ were significantly higher than for the previous simulations, indicating a deteriorated fit to the histogram. There were at least two local minima in $\chi^{2}$, with about equal values, represented by the two curves in the figure. The uncertainties in the derived numbers of stars are large, even for stars of magnitude 20 .

\subsection{Other shapes for the $L F$}

The LF in the simulation above increased linearly in a logarithmic plot. Simulations with a turned-down and a turned-up LF were made to see if LuMINous could derive the LF also in these cases. The results for the simulations are given in Fig. 7 .

For the turned-down LF, the general appearance of the smoothed derived LF seems correct, with the exception of some small fluctuations for the highest magnitudes. The other simulation, with the turned-up LF and higher crowding than the one with the straight LF, showed some larger fluctuations around magnitude 21. The smoothed derived LF followed the general features of the input LF reasonably well for the faintest magnitudes.

\subsection{Extreme crowding}

Both spatial (pixel) and intensity (gain) sampling were poor for the simulation discussed above. To see how the sampling affects the results, simulations were made with changed spatial and intensity resolution, again using the LF from Fig. 4.

For ground based images of crowded fields the main limitation for detection of fainter stars is the confusion problem, not the signal to noise ratio with respect to the background noise. Figure 8 shows the LFs derived from

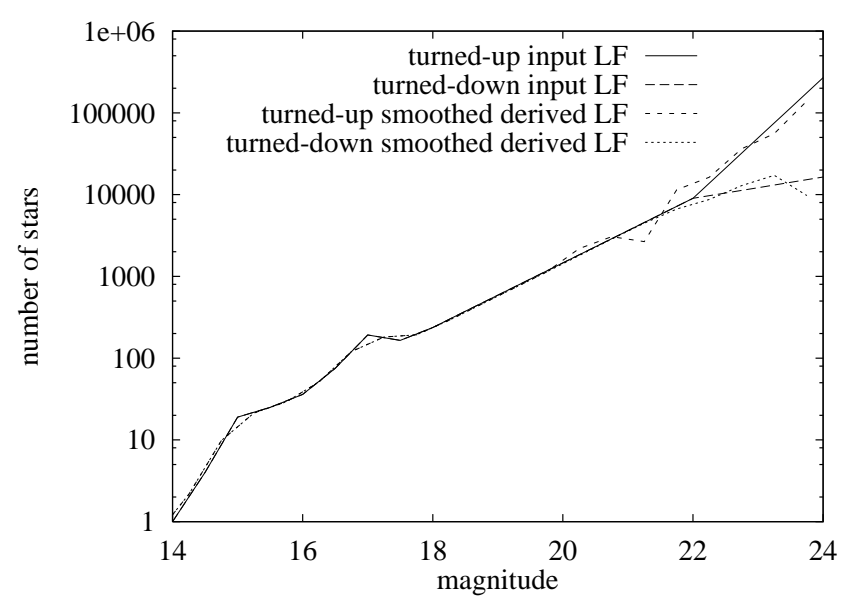

Fig. 7. LFs for simulated images with a steeper or lower slope in the faint part of the LF

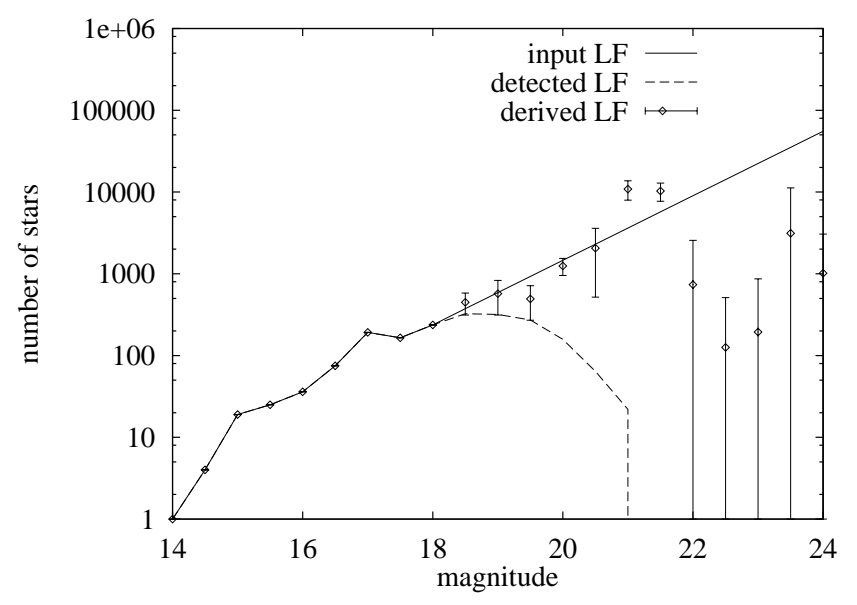

Fig. 8. Input, detected and derived LF for an image with a PSF with large FWHM, resulting in extreme crowding. Estimates for the uncertainties in the derived LF are indicated by the error bars, for solutions with $\chi^{2}<1.10$

a simulated image with a well sampled PSF (FWHM 11 pixels), together with the LF of the stars detected with DAOPHOT. Changes of crowding were made through increment of the FWHM of the PSF, while leaving the positions and relative intensities of the stars unchanged. The change in the PSF did not conserve flux. The stars in the simulation with the wider PSF had higher intensity, and thus, with all other parameters unchanged, a higher signal to noise ratio. The incompleteness of detection was strictly due to crowding effects. The uncertainty in the number of stars in each magnitude bin could, just as in the previous simulations, be approximated with an exponential function of the magnitude.

As seen from Fig. 8, the uncertainty in the derived LF became larger than the derived value for magnitude 22.0 and higher. A change in the number of stars for these 
magnitudes could not be discerned from a change in the diffuse background level.

\subsection{Well sampled read-out noise}

The intensity sampling of the simulated image was changed from 7 electrons per ADU to 1.7. When expressed in electrons, the intensities of the stars and amplitude of the read-out noise were unchanged. The derived LF was smoother in appearance than for the simulation with gain 7. When smoothed, the derived LF was very close to the input LF. The estimated uncertainties in the numbers of stars in the derived LF were similar to the uncertainties for the simulation with gain 7 .

\section{An application}

A number of HST WFPC2 exposures were made of the central part of the LMC Bar for an ongoing investigation of the chemical composition and star formation history of the Large Magellanic Cloud (LMC) (Ardeberg et al. 1997). Among those, a total of 16 Strömgren y exposures of the same field are available. A single exposure was used to test the method on a real image. The combined exposures allowed the LF to be measured with classical methods to a fainter limit than possible in a single exposure. This way, the results of LUMINOUs were checked against the LF determined from counts of individually measured stars of the same field, serving as the "true" LF. For this exposure, the detection of faint stars is mainly limited by the signal to noise ratio of the stars, not the degree of crowding. The simulations in the previous section are similar to the HST exposure, both in crowding and noise properties.

\subsection{Validity of the assumptions}

The assumptions mentioned in Sect. 2.3 are not completely fulfilled. The test with the WFPC2 image shows the combined effect of the errors in the assumptions.

Only stars are present. DAOPHOT photometry of the combined images showed that nearly all objects could be described well using star-like objects. Visual inspection suggested the same.

Distribution of stars. The visible stars were distributed randomly, the field of view was small and located in the centre of the LMC. There was no reason to assume any special distribution for the faint stars. Any clustering of faint stars near bright ones was assumed absent.

Constant PSF. This assumption was not correct. The PSF varies over the image (Linde \& Snel 1997; Biretta et al. 1996). The current version of the software used did not allow for a variable PSF. However, the LF derived from the central $25 \%$ of the image did not differ from that derived from the central $60 \%$.

Read-out noise. Investigation of WFPC2 bias images showed that the read-out noise could accurately be described by a Gaussian distribution. Since the read-out noise was about $0.7 \mathrm{ADU}$, few bins with information about the shape of the noise distribution were available.

Diffuse sky background. Visual inspection of the image showed no gradients or large scale structure in the sky background.

\subsection{Calibration files}

The pipeline calibration of WFPC2 images corrects for a large number of effects (Biretta 1996). Standard calibration files for these effects are available in the HST archive, and were used for the simulation of the effects as well. These included modelling of effects of the flat field, darkimage and bias-image.

A variable effect of the bias image has been observed (Holtzman et al. 1995), resulting in different bias levels for the odd and even columns in the image. These levels, and the difference between them, differ from exposure to exposure. This odd-even effect has been included in the model.

\subsubsection{Analog-Digital Converter effects}

Measured ADC effects for WFPC2 are available from the HST data archive. There has been no demand for an accurate description of these effects, so no update of the information has been made (Stiavelli 1997). The standard ADC effect calibration file from the Space Telescope Science Institute was modified with the help of exposures from the HST archive, and used to model the effect.

\subsubsection{Bias jumps}

WFPC2 images can contain bias jumps, changes in the bias level within a single image. Linde \& Snel (1997) have detected small bias level jumps in WFPC2 images, undetectable without heavy smoothing to suppress read-out noise. Such effects, if present in the image, would have the same effect as an error in the bias image, or an increase in the read-out noise, combined with a change in the distribution of the read-out noise.

\subsection{LMC Bar results}

The LF of the images was determined in four different ways: direct detection in a single exposure, direct detection in an average of 16 exposures, derived with LUMINOUS from a single exposure using a 4.5 pixel PSF radius, and using a Tiny Tim (Krist 1995) model PSF (Fig. 9).

The LF from the averaged image was corrected for incompleteness and was used to check the validity of the derived LF. The correction was done by estimating completeness of detection using artificial stars. The detected LF from the single image was also corrected for incompleteness and is shown as a comparison in Fig. 9.

Both solutions using LUMINOUs were sensitive to the adopted value of the read-out noise. This value was adjusted to obtain lowest $\chi^{2}$, and was within one sigma of the value tabulated by Biretta (1996). For both the extracted and model PSF it was possible to obtain a $\chi^{2}$ of 


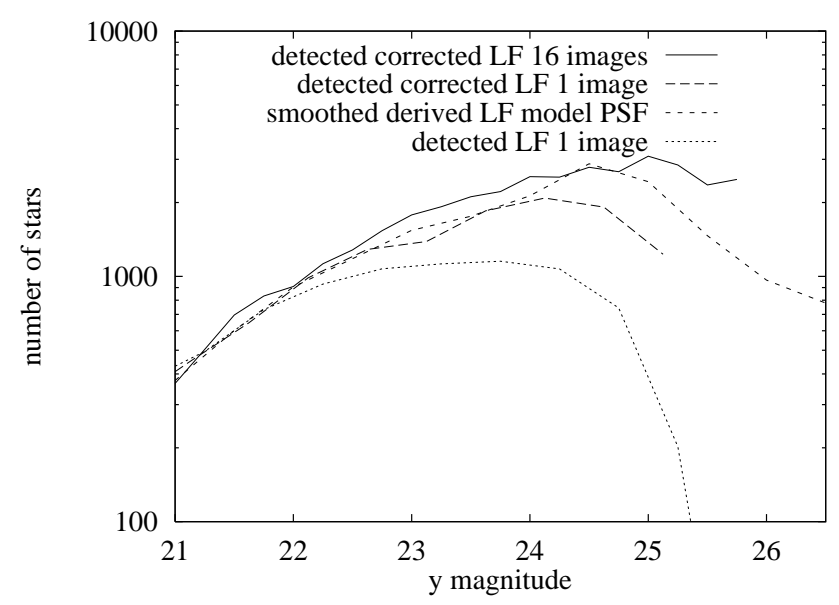

Fig. 9. LFs measured in an average of 16 WFC exposures of the LMC Bar and corrected for completeness, measured in a single WFC exposure and corrected for completeness, smoothed derived LF for a single exposure using a model PSF, and measured in a single WFC exposure

about 1.2. The corresponding LFs were nearly identical for all but the faintest magnitudes, but deviated somewhat from the LF derived from the average image. The slightly high value for $\chi^{2}$ indicated that the fit to the histogram was less than optimal.

\section{Discussion}

Information about the distribution of point sources on the sky can be derived in a number of ways. The methods can be divided in those that detect individual stars and those that use a statistical description of the distribution of sources, without the need to detect individual sources. The magnitude limit for individual detections is given by Saha (1995), and is dependent on the allowed rate of spurious detections. Zepka et al. (1994) use information from the statistics of the noise to reduce the rate of spurious detections. Analysis of the probability density function of the observed intensities is in use in radio (Condon \& Dressel 1978) and X-ray (Barcons 1992) astronomy. The intensity distribution of sources can be derived to source densities of about one source per beam (Scheuer 1974). LuMinous falls in the same category as probability density analysis.

Detection of individual stars is an accurate method for determining the LF, as long as the stars can easily be detected and measured. When the completeness of detection becomes too small, probability density analysis yields better results.

\subsection{Uniqueness of the solution}

Solutions obtained with LUMINous should be checked for uniqueness. Both in generating the image from the LF and in generating the histogram from the image, information can potentially get lost or distorted. The correlation between the number of stars of a certain magnitude and the intensity of the background determines the limiting magnitude. As the correlation coefficient approaches -1, it becomes impossible to discern between adding a number of stars or increasing the diffuse background. Likewise, the correlation coefficient between two adjacent magnitude bins in the LF can approach -1 , which makes them indiscernible. As seen from the derived LF in Fig. 4, there were some large fluctuations in the faint part of the LF. Smoothing effectively removed the fluctuations, leaving the LF close to the input one. The effect of smoothing on $\chi^{2}$ was negligible, indicating that the unsmoothed and smoothed solutions were equivalent. The derived solution was not unique. But since it is unlikely that the fluctuations are physical, the smoothed LF is preferred.

\subsection{The PSF}

All stars in the simulated image have the same shape, the PSF. Errors in the PSF affect the derived LF. When the PSF is measured directly from the image, there may be several faint, undetected stars present within the radius of measurement. The shape of the analytical part of the PSF may contain errors (e.g. the wings are too bright or faint). In practical cases, a perfect PSF cannot be obtained. Noise in the PSF can affect the noise in the background and change the appearance of the histogram. Care should therefore be taken when extracting the PSF from an image.

The simulations for which the PSF was extracted from the image (Fig. 5) show that the choice of a large PSF radius was not the best. The smaller PSF gave a better derived LF, despite the fact that part of the information in the wings was discarded.

\section{3. $A D C$ effects}

In classical CCD photometry it is customary to correct for sensitivity variations of the detector with a flat fielding procedure. This is of significant importance for the accuracy of the photometry. LUMINOUS does not operate on the image, but on the histogram of the image. To achieve best results, one must correct for variations in the histogram bin width. An ADC does the actual binning of electron counts into discrete ADU values. However, this conversion is not perfect: both non-linearity effects and variable bin width affect the appearance of the histogram. The effect on classical photometry is usually negligible. When trying to adjust the histogram of a simulated image to that of an observed image, accurate modelling of the ADC effects is of similar importance as the flat field is for photometry. The simulation with the ADC errors (Fig. 6) showed that large systematic errors in the derived LF can arise, even for relatively bright stars. In the first applications of LUMINOUS to the WFPC2 image, the fit to the histogram of the image was not good in some bins. This resulted in both a large $\chi^{2}$ and large systematic errors in the derived LF. It is of importance to correctly incorporate the ADC effects, specifically for those intensity bins that contain the 
majority of the pixels in the image. Suitable calibration images for characterisation of the effect could e.g. have a flat histogram of pixel intensities, such as a linear intensity ramp in the image.

\subsection{Expected accuracy}

As can be seen from the uncertainties in the numbers of stars in the simulation (Fig. 8), the faintest part of the derived luminosity function had large uncertainties. The uncertainty in the derived LF grew approximately exponentially with magnitude, with an exponent larger than the exponent of the input LF. The actual values of the uncertainties increased with the degree of crowding. The uncertainties were determined from the spread of the derived LF parameters for solutions having a $\chi^{2}$ lower than a fixed value.

The solutions were checked against Scheuer's (1974) limit of one source per beam. The area of the beam was calculated as $\pi \mathrm{FWHM}^{2} / 4$ pixels, FWHM being the full width at half maximum of the PSF. The size of the image was $800 \times 800$ pixels. With the PSF, ADC effects, flat field, read-out noise, bias level, gain and location and intensities of the detectable stars perfectly known, the derived LF was composed of approximately 0.4 sources per beam for $\mathrm{FWHM}=1.5$, and about four sources per beam for $\mathrm{FWHM}=11$. The order of magnitude of detected source density is correct, both for the case of background and read-out noise limited detection $(\mathrm{FWHM}=1.5)$ and confusion limited detection $(\mathrm{FWHM}=11)$. The ratio of the number of directly detectable stars versus derived number of stars was in both cases about $1: 10$.

If the detected LF was truncated at the magnitude where completeness of detection dropped below 0.5 , and the derived LF at the magnitude where the uncertainty in the LF exceeded the star count, LUMINOUs could measure the LF about 2 magnitudes deeper.

Systematic errors could limit this range somewhat. Errors in the bias level and read-out noise affected mainly the counts for the faintest 1.5 magnitudes of the LF. The error in the read-out noise was of importance, indicating that an accurate value for the read-out noise is desirable. However, knowledge of the ADC effects was of much greater importance. Errors in the simulation of the ADC effects caused systematic errors for the counts of stars that were bright enough to be individually detected.

The sampling of the intensities in the image had no significant effect on the accuracy. It was possible to retrieve an LF with either an increasing or decreasing slope. The shape of the LF affected the accuracy through the limit of one source per beam, causing a somewhat larger uncertainty for a steeper LF. The crowding of the image, changed by increasing the FWHM of the PSF, caused a larger uncertainty for higher crowding, as could be expected from the source density limit. However, the number of sources per beam increased to four, indicating that
LUMINOUS effectively derived the LF, despite the fact that a lower limiting magnitude was reached.

In the LMC Bar exposure with the Wide Field Camera of WFPC2 it was possible to reach about 1.5 magnitudes deeper than direct detection and obtain reproducible results, but with systematic errors. The fit was not optimal, judged by the $\chi^{2}$ value of 1.2 . The cause of this error is most likely a combination of effects, some of which have been discussed above. Since the LFs derived with the measured and modelled PSF are so similar, and considering the simulations with the PSF errors, it is unlikely that the errors in the PSF are dominating the systematic errors. A likely candidate for these systematic errors is the ADC correction. The simulations have shown that large systematic errors can arise due to ADC correction errors. Because of the coarse intensity sampling, only a few histogram bins are effectively used to derive information about the LF. A modelling error affecting one or two of these bins will have large impact on the LF. Another possible error source is the assumption about the shape of the distribution of the read-out noise: $\chi^{2}$ was sensitive to the width of the assumed Gaussian distribution. If the actual distribution was not Gaussian (bias jumps can widen the distribution), this would affect the fit and the derived LF. A better intensity sampling would allow for a better determination of the read-out noise characteristics.

The deviations between the derived LF from the single exposure and the detected LF from the averaged image, together with the less than optimal fit to the histogram, stress the importance of calibration of the effects that contribute to the shape of the histogram. Accurate calibrations of the ADC effects and shape of the read-out noise would allow for more accurate modelling, reducing the systematic errors due to these effects. High resolution sampling of the intensities should facilitate the determination of these effects.

\subsection{Possible improvements}

A point where Luminous can be improved is the measure of similarity used. The histogram of the image was chosen because it contains no information about the exact location of the stars. The information is not available from the image, and not necessary for derivation of the LF. Comparison between the observed and simulated image must be done with some measure that is insensitive to the location of the stars. The texture of the image could be such a measure of similarity.

A histogram of pixel intensities of the image loses all information about neighbouring pixels. Instead of taking a one dimensional histogram, a second dimension can be added, describing the surroundings of the pixel in question. If a simple average intensity of the eight surrounding pixels is taken as a second dimension, a new sort of histogram is obtained. For a well sampled image, the intensities of the pixels of stars $\left(I_{\mathrm{p}}\right)$ are correlated with the intensities of their surrounding pixels $\left(I_{\mathrm{S}}\right)$. This means that 
the major part of the pixels will end up near the diagonal of the histogram, where $I_{\mathrm{p}}=I_{\mathrm{s}}$. CREs, hot pixels, image defects, etc will generally have little correlation with their surroundings, and do not adhere to the diagonal. Using the two dimensional histogram of the image as a measure of similarity may improve LuMINOUS. An extra advantage of the two dimensional histogram is that changes in the width of the PSF are reflected in the degree of correlation, or the spread around the diagonal.

The derived LF fluctuated with large gradients around the input LF. An additional constraint for the derived LF could be one of smoothness, using a maximum entropy method to adjust the number of stars in the LF bins.

The simulated image should be as similar as possible to the observed image. A choice must be made if an effect is important enough to be put in the simulation, and if it is known well enough to be modelled instead of assuming some generic description. A more careful description of the various aspects responsible for the appearance of the image could prevent systematic errors in the derived LF.

The problem of finding the best LF to describe the image is one of minimisation. Like with any such problem, it is essential to use a good algorithm to find the minimum in $\chi^{2}$ space. A better algorithm may be able to find a better minimum, possibly with a better LF.

\section{Conclusions}

- With Luminous, it is possible to derive the faint part of the luminosity function to the limit of approximately one source per beam.

- The derived solution is not unique, but smoothing the derived LF produces acceptable results.

- When compared with the limit of detection for classical photometry (DAOPHOT was used as a comparison), the derived LF contains about ten times as many stars as the directly detected LF, or when expressed in limiting magnitude, up to two magnitudes fainter for the LF used in this simulation.

- The sampling of the intensities in the image has no effect on the limiting magnitude or uncertainty in the LF, but affects smoothness of the derived LF.

- The uncertainty in the LF grows exponentially with stellar magnitude, faster than the LF, defining an upper limit on the magnitude. The accuracy is dominated by the accuracy of the models of the ADC effect, PSF and read-out noise. The shape of the LF has no significant effect on the accuracy.

Provided that accurate calibrations of the dominating sources of uncertainty are available, LUMINOUs is an excellent complement to classical photometry, allowing the LF to be measured up to two magnitudes fainter, at no additional observational cost.

Acknowledgements. I thank Massimo Stiavelli at Space Telescope Science Institute for many useful discussions on various kinds of noise sources for the WFPC2.

\section{References}

Ardeberg A., Gustafsson B., Linde P., Nissen P.-E., 1997, A\&A 322, L13

Barcons X., 1992, ApJ 396, 460

Biretta J.A., Burrows C., Holtzman J.A., et al., 1996, WFPC2 Instrument Handbook, vers. 4.0, STScI, Baltimore

Condon J.J., Dressel L.L., 1978, ApJ 222, 745

Holtzman J.A., Hester J.J., Casertano S., et al., 1995, PASP 107,156

Krist J., 1995, Simulation of HST PSFs using Tiny Tim. In: Shaw R.A., Payne H.E., Hayes J.J.E. (eds.), Astronomical Data Analysis Software and Systems IV, ASP Conf. Ser. 77,349

Linde P., Snel R., 1997, Towards Optimal Analysis of HST Crowded Stellar Fields. In: Hunt G., Payne H.E. (eds.), Astronomical Data Analysis Software and Systems VI, ASP Conf. Ser. 125

Saha P., 1995, AJ 110, 916

Scheuer P.A.G., 1974, MNRAS 166, 329

Stetson P.B., 1987, PASP 99, 191

Stiavelli M., 1997 (private communication)

Zepka A.F., Cordes J.M., Wasserman I., 1994, ApJ 427, 438 